\title{
Impacto de multimorbidade sobre mortalidade em idosos: estudo de coorte pós-hospitalização
}

\author{
Impact of multimorbidity on mortality in elderly: a post-hospitalization cohort study
}

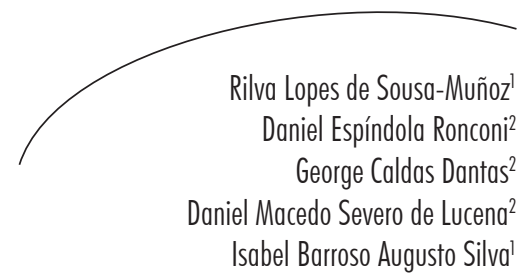

Resumo

Objetivos: Avaliar a associação entre comorbidades e risco de morte e reinternação em idosos após alta de enfermarias de clínica médica de um hospital universitário. Métodos: Estudo de coorte de idosos que receberam alta do Hospital Universitário Lauro Wanderley. Foram utilizados o Índice de Comorbidade de Charlson (ICC) e o ICC associado à idade (ICIC). O desfecho primário foi mortalidade pós-hospitalização e o secundário, ocorrência de

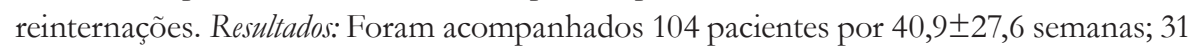
$(29,8 \%)$ morreram e 38 (36,5\%) foram reinternados. A curva de sobrevida foi descendente com proporção acumulada de $50 \%$. Óbito relacionou-se com idade $(\mathrm{p}=0,04)$, número de prescrições hospitalares ( $\mathrm{p}=0,01), \operatorname{ICC}(\mathrm{p}=0,001)$ e ICIC $(\mathrm{p}=0,001)$. Não houve associação de ICC com reinternação. Conclusões: A gravidade das comorbidades em idosos relacionouse com maior risco de morte pós-hospitalização. Salienta-se a necessidade de se disporem de dados de comorbidade para avaliar cuidados a essa clientela.

\section{Abstract}

Objectives: To evaluate the association between comorbidities and risk of death and readmission after discharge of elderly after discharge from medical wards in a university hospital. Methods: A cohort study of elderly patients discharged from University Hospital Lauro Wanderley. We used the Charlson Comorbidity Index (CCI) and the ICC associated with age (ICIC). The primary outcome was post-hospitalization mortality ; the secondary, readmissions. Results: 104 patients were followed for $40.9 \pm 27.6$ weeks; 31 $(29.8 \%)$ died and 38 (36.5\%) were readmitted. The survival curve was descending with cumulative proportion of $50 \%$. Death was related to age $(\mathrm{p}=0.04)$, number of hospital prescriptions $(\mathrm{p}=0.01), \mathrm{CHF}(\mathrm{p}=0.001)$ and ICIC $(\mathrm{p}=0.001)$. There was no association of ICC with rehospitalization. Conclusions: The severity of comorbidities in the elderly was related to increased risk of death after hospitalization. This emphasizes the need to dispose of comorbidity data to evaluate care for these patients.

\footnotetext{
Departamento de Medicina Interna, Centro de Ciências Médicas. Universidade Federal da Paraíba. João Pessoa, PB, Brasil.

2 Curso de Medicina, Centro de Ciências Médicas. Universidade Federal da Paraíba. João Pessoa, PB, Brasil. Pesquisa financiada com bolsa do Programa Institucional de Bolsas de Iniciação Científica CNPq/UFPB.
}

Palavras-chave: Idoso. Comorbidade. Mortalidade.

Key words: Elderly. Comorbidity. Mortality. 


\section{INTRODUÇÃO}

A comorbidade é um importante preditor de complicações e resultados desfavoráveis em doentes idosos, principalmente quando hospitalizados. ${ }^{1,2} \mathrm{O}$ peso dos diagnósticos secundários (denominados comorbidades) na gravidade dos pacientes varia segundo o diagnóstico principal, mas certas combinações de comorbidades apresentam maior risco que outras. ${ }^{3} \mathrm{O}$ efeito da multimorbidade sobre a mortalidade, portanto, deve ser avaliado não apenas pela quantidade de doenças coexistentes, mas também por sua natureza e gravidade. ${ }^{4}$

A abordagem da comorbidade é mais uma dessas áreas de incerteza em que os médicos aplicam o raciocínio hipotético-dedutivo baseado na experiência adquirida com a prática clínica. ${ }^{5}$ Há pouco publicado na literatura médica sobre esse tema, e doentes portadores de múltiplas doenças crônicas são frequentemente excluídos de estudos clínicos e, por consequência, há pouca informação disponível sobre esse grupo de pacientes. ${ }^{6-9}$

A hipótese de pesquisa do presente estudo foi que o número e a gravidade de comorbidades do paciente idoso egresso de hospitalização em enfermarias de clínica médica de um hospital universitário são determinantes de maior mortalidade e reinternações no período de até dois anos após a hospitalização. O objetivo foi verificar o índice de gravidade e o número de comorbidades como fator preditivo de óbito e reinternação da clientela de idosos egressa de hospitalização das enfermarias de clínica médica do Hospital Universitário Lauro Wanderley (HULW).

\section{METODOLOGIA}

\section{Modelo do estudo}

Estudou-se uma coorte de pacientes idosos que receberam alta hospitalar do Serviço de Clínica Médica do HULW, situado na cidade de João Pessoa, Estado da Paraíba, Brasil, entre agosto de 2008 e setembro de 2010, incluindo as censuras e óbitos.
Amostra

A amostra foi composta inicialmente por 147 pacientes, recrutados por conveniência e que receberam alta das enfermarias de clínica médica (ECM) do HULW, com seguimento até dois anos depois do início da coleta de dados. Foram incluídos os pacientes com idade igual ou superior a 60 anos, de acordo com o critério cronológico adotado pela Política Nacional de Saúde do Idoso (PNSI) do Ministério da Saúde para definição operacional de indivíduo idoso. ${ }^{10}$

\section{Critérios de elegibilidade}

Foram adotados os seguintes critérios de inclusão: a) paciente admitido para tratamento clínico nas ECMs do HULW; b) idade maior ou igual a 60 anos; c) consentimento informado para participação na pesquisa. Foram excluídos da amostra os pacientes que se recusaram a participar do projeto, assim como os que apresentaram permanência hospitalar menor que dois dias (período de tempo arbitrariamente considerado insuficiente para avaliar a evolução clínica) e óbitos antes da alta hospitalar.

O recrutamento foi realizado observandose os critérios de inclusão e exclusão do estudo, com o comparecimento de três estudantes de Medicina previamente treinados nas enfermarias, onde existem 70 vagas disponíveis para internamento de pacientes adultos, realizando-se contatos com os médicos residentes do serviço para verificação das previsões de alta dos sujeitos elegíveis do estudo.

\section{Procedimentos de coleta de dados}

A abordagem inicial dos pacientes foi feita no dia da alta, e o acompanhamento posterior foi ambulatorial (ambulatório de egressos de internação das ECMs do HULW) e domiciliar, por contato telefônico com os que deixaram de comparecer às visitas agendadas. Foi feita também a captação de dados referentes ao período prévio à alta (período de hospitalização), por meio da 
revisão dos prontuários e de entrevistas com os pacientes e seus familiares. No dia da alta, foi feito o agendamento para retorno ao ambulatório dos pacientes incluídos no estudo dez semanas após a alta hospitalar, quando foram reavaliados pelos estudantes, sob supervisão médica.

Os dados primários foram obtidos por meio de entrevistas padronizadas com pacientes e familiares acompanhantes (variáveis demográficas; preenchimento do Índice de Comorbidade de Charlson; doenças atuais diagnosticadas; causa da internação; número de hospitalizações no último ano; acompanhamento após alta hospitalar com datas; número de visitas ambulatoriais; número de contatos telefônicos; estado do paciente em cada um dos contatos pósalta; desfechos). Os familiares foram interrogados quando os pacientes não estavam em condições de se comunicar.

\section{Variáveis independentes}

Os fatores de risco que, na hipótese desta pesquisa, se relacionam com mortalidade dentro de um período de até dois anos, foram agrupados nas seguintes categorias: Índice de Comorbidade de Charlson, número de comorbidades, outras variáveis clínicas e variáveis demográficas. Os diagnósticos foram agrupados por categorias de doenças, segundo a $10^{\mathrm{a}}$ Classificação Internacional de Doenças. ${ }^{11}$ As categorias foram consideradas na análise quando sua prevalência na amostra atingiu mais de $10 \%$.

As variáveis demográficas e clínicas foram registradas em formulário padronizado elaborado pelos autores, a saber: (a) demográficas: idade (estratificada em: 60-69, 70-79, 80 ou mais); sexo; estado civil; grau de instrução; procedência (capital/interior); renda familiar; ocupação; número de pessoas que moram com o paciente; e (b) clínicas: diagnóstico definitivo principal (problema clínico que motivou a internação do paciente, como registrado na história clínica de admissão no hospital e corroborado pelo diagnóstico de alta); número de comorbidades (doenças concomitantes à doença principal); número de atendimentos médicos e hospitalizações no ano anterior à atual internação; permanência hospitalar (número de dias de internação).

\section{Variáveis dependentes}

O desfecho primário da pesquisa foi a variável dicotômica mortalidade (sim/não). O desfecho secundário foi reinternação, também binária ( $\operatorname{sim} /$ não). As variáveis idade e quantidade de comorbidades foram tratadas como contínuas e o Índice de Comorbidade de Charlson (ICC) foi agrupado nas categorias 1, 2 e 3 ou mais.

Instrumentos de coleta de dados

Para avaliar a gravidade das comorbidades, foi utilizado o Índice de Comorbidade de Charlson (ICC) e posteriormente esse mesmo índice associado à idade (ICIC). O Índice de Charlson é um método de classificação de gravidade que utiliza dados dos diagnósticos secundários para atribuir um risco de morte ao paciente, empregado em diversos trabalhos, e recentemente também no Brasil. ${ }^{12-16}$ Esse método é aplicado por meio de um software com uma lista de 19 condições clínicas registradas como diagnóstico secundário, com o objetivo de medir a gravidade e avaliar seu efeito sobre o prognóstico do paciente. ${ }^{17}$ Para cada uma dessas condições, é atribuída uma pontuação com base no risco relativo, gerando os pesos $0,1,2$ e 3 . Para gerar um escore único paralelamente, a idade do paciente também tem um peso: a partir dos 50 anos, cada período de dez anos corresponde a um ponto adicional no índice.

Os casos foram separados de acordo com o diagnóstico principal, e foram aplicados os respectivos pesos em cada caso, conforme o número e gravidade dos diagnósticos secundários e, posteriormente, o Índice de Charlson combinado, ou seja, o ICIC. O ponto de corte de 5 ou mais na pontuação do ICC foi usado de acordo com estudos prévios. ${ }^{1,18}$ 
Análise estatística

A análise estatística realizada foi baseada em medidas de associação de risco (risco relativo ou hazard ratio). A análise de sobrevida foi realizada com a construção de curvas de pacientes vivos, segundo a técnica Kaplan-Meier. ${ }^{19}$ Os pacientes que não completaram o seguimento de dois anos foram censurados na última informação (visita ambulatorial ou contato telefônico). ${ }^{20} \mathrm{O}$ teste $t$ de Student também foi usado para as variáveis contínuas com distribuição paramétrica; o teste de Mann-Whitney foi usado para as variáveis com distribuição não paramétrica; e o teste de quiquadrado (e o teste exato de Fisher), para dados categóricos. Adotou-se o nível de significância de $5 \%$. Essas análises foram realizadas por meio do programa estatístico SPSS, versão 17.0.

\section{Aspectos éticos}

Após aprovação do projeto pelo Comitê de Ética em Pesquisa do HULW (CEP-HULW), protocolo $n^{\circ}$ 006/2008, os pacientes elegíveis foram convidados a participar da pesquisa e assinaram o Termo de Consentimento Livre e Esclarecido aprovado pelo CEP.

\section{RESULTADOS}

No período de recrutamento da amostra, foram incluídos 147 pacientes idosos hospitalizados no Serviço de Clínica Médica do HULW. Destes, 19 morreram antes da alta; 13 solicitaram saída do estudo; dez residiam em áreas distantes da cidade de João Pessoa e um recebeu alta hospitalar a pedido. Foram seguidos 104 pacientes $(70,7 \%$ da amostra inicial), acompanhados pelo período de uma a 94 semanas $(40,9 \pm 27,6) ; 31$ pacientes deixaram a coorte pelo desfecho óbito, ocorrido de uma a 64 semanas após a alta hospitalar $(24,3 \pm 20,7)$.

No período de observação de até dois anos, foram programados contatos com os pacientes e/ ou suas famílias, agendados para 10, 20, 30, 40 e 50 semanas após a alta hospitalar.
A idade mínima da amostra recrutada e seguida foi de 60 anos, e a máxima, de 98 anos, com média de $69,5( \pm 7,7)$. Classificando-se a amostra por faixas etárias, $56(53,8 \%)$ tinham entre 60 e 69 anos; 37 tinham entre 70 e 79 anos (35,6\%); nove $(8,7 \%)$ tinham entre 80 e 89 anos e dois $(1,9 \%)$ tinham mais de 90 anos. Quanto ao gênero, 58 pacientes eram do sexo masculino (55,8\%). Em relação à etnia, 58 pacientes $(55,8 \%)$ eram pardos, $31(29,8 \%)$ brancos e $15(14,4 \%)$ negros.

Houve equivalência quanto à procedência dos pacientes, classificada em oriundos da capital (cidade de João Pessoa e região metropolitana) $(49 / 47,1 \%)$ e aqueles vindos do interior do Estado da Paraíba (55/52,9\%).

O número de consultas médicas realizadas durante os últimos 12 meses variou de 0 a 35 $(7,6 \pm 7,0)$, e o número de hospitalizações no mesmo período variou de 0 a 7 hospitalizações, excluindo-se a atual $(1,3 \pm 1,6)$. $O$ número de prescrições medicamentosas simultâneas previamente à hospitalização atual foi de 0 a 8 $(1,9 \pm 3,5)$ por paciente, enquanto o número de medicamentos simultaneamente prescritos durante a hospitalização variou de 0 a $13(5,9 \pm 2,2)$.

Quanto ao diagnóstico principal por enfermidade específica, insuficiência cardíaca congestiva e neoplasias foram os diagnósticos mais frequentes, configurando a frequência relativa de 45,1 , seguidos por doenças pulmonares e hepáticas crônicas.

Dos pacientes avaliados, 92,3\% (96/104) receberam alta com melhora clínica de seu quadro, enquanto $7,7 \%$ (8/104) foram transferidos da enfermaria de clínica médica para outros setores do hospital ou para outra instituição. O tempo de permanência hospitalar variou de quatro a 92 dias (média: 30,8土18,2).

Trinta e um pacientes $(29,8 \%$ ) morreram após a alta hospitalar em seu domicílio e 38 pacientes $(36,5 \%)$ foram reinternados no mesmo serviço (ECM do HULW) ou em outra instituição. Dos pacientes reinternados, 20 reinternaram-se uma única vez; oito pacientes foram reinternados duas vezes; dois pacientes reinternaram-se três 
vezes e um paciente, quatro vezes, durante o período de acompanhamento.

A figura 1 mostra resultados da análise de sobrevida com taxas cumulativas de mortalidade de acordo com a técnica Kaplan-Meier. ${ }^{19}$ A média do tempo de sobrevivência foi de 23,8 $\pm 1,6$ semanas (IC 95\%: 20,6-26,9). Verificou-se que a curva de sobrevida foi descendente, observandose que a proporção acumulada de sobrevida foi de apenas 50\%, 20 semanas após a alta hospitalar.

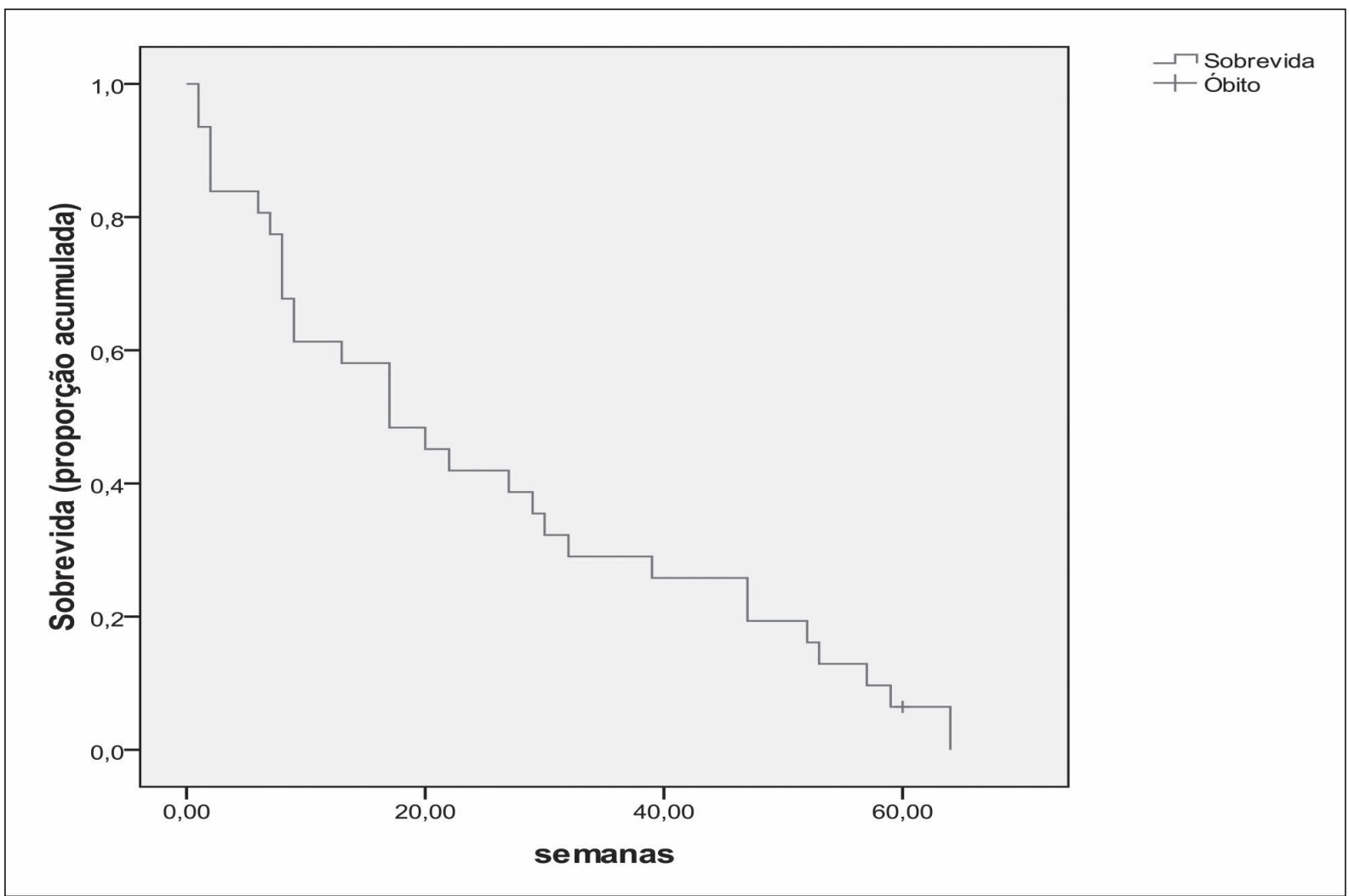

Figura 1 - Estimativa da probabilidade de sobrevida com construção de curva de pacientes vivos, segundo a técnica Kaplan-Meier, dos pacientes idosos que receberam alta hospitalar do Serviço de Clínica Médica no período de 11 meses, com acompanhamento por um período de uma a 44 semanas $(21,6 \pm 12,2)$ (n=61). João Pessoa-PB, 2008-2010.

A pontuação do ICC variou de 0 a 8 na amostra $(2,7 \pm 2,1)$ e o ICIC, de 2 a 12 pontos $(5,3 \pm 2,2)$. A presença de uma ou mais comorbidades, definida como a coexistência de outras doenças além da principal em um mesmo indivíduo (analisada como variável binária $\operatorname{sim} /$ não ou contagem simples), foi observada em 92,3\% (96/104) dos pacientes. Hipertensão arterial, diabetes mellitus tipo-2, insuficiência cardíaca congestiva, doença pulmonar obstrutiva crônica e hepatopatia crônica foram as comorbidades mais frequentes. O número de comorbidades variou de 0 a 6 , com média de $2,1( \pm 1,23)$.

Houve associação estatisticamente significativa entre presença de comorbidade (variável binária: $\operatorname{sim} /$ não) e sexo dos pacientes $(\mathrm{p}=0,03)$, sendo aquela variável mais frequente no sexo feminino. 
A presença de comorbidade ocorreu em 100\% das mulheres da amostra e em 84,4\% dos homens.

A presença de comorbidade não se relacionou com as demais variáveis sociodemográficas pesquisadas (etnia, procedência e atividade laborativa atual), nem com as variáveis clínicas (duração da permanência hospitalar; etilismo; tabagismo; número de atendimentos médicos no último ano; número de internações anteriores; número de internações no último ano; número de medicamentos prescritos antes da hospitalização e número de medicamentos usados no momento da alta hospitalar). Os escores do ICC também não variaram estatisticamente de acordo com as referidas variáveis explanatórias (tabela 1).

Tabela 1 - Valores médios e desvios-padrão do Índice de Comorbidade de Charlston (ICC) em relação às variáveis sociodemográficas $(\mathrm{n}=104)$. Enfermarias de Clínica Médica do Hospital Universitário Lauro Wanderley. João Pessoa-PB, 2008-2010.

\begin{tabular}{llccc}
\hline & Variável & Média ICC & $\begin{array}{c}\text { Desvio-padrão } \\
\text { ICC }\end{array}$ & $\mathrm{p}$ \\
\hline Idade & $60-69$ & 2,6 & 1,6 & \\
& $70-79$ & 2,5 & 2,5 & NS \\
Gênero & $\geq 80$ & 3,7 & 3,4 & \\
& masculino & 2,5 & 1,7 & NS \\
Etnia & feminino & 3,3 & 2,5 & \\
& branca & 3,3 & 2,6 & NS \\
& parda & 2,7 & 2,0 & NS \\
Procedência & negra & 2,8 & 1,7 & \\
& João Pessoa & 2,4 & 1,1 & NS \\
Atividade laboral & interior da PB & 2,1 & 2,0 & \\
& ativo & 2,9 & 2,1 & \\
& inativo & 2,9 & 2,1 & \\
\hline
\end{tabular}

ICC: Índice de Comorbidade de Charlston; PB: Paraíba; p: probabilidade de significância estatística;

NS: sem diferença estatisticamente significativa entre os grupos.

Por outro lado, houve associação estatisticamente significativa entre idade e óbito $(\mathrm{p}=0,04)$, verificando-se idade maior entre os pacientes que morreram que nos que permaneceram vivos no período de observação.

Não houve associação estatisticamente significativa entre ocorrência de óbito ou reinternação com as variáveis sociodemográficas (sexo, etnia, procedência, atividadelaborativa atual) e clínicas (duração da permanência hospitalar; presença de comorbidade [contagem simples]; etilismo; tabagismo; número de atendimentos médicos no último ano; número de internações anteriores; número de internações no último ano; número de medicamentos prescritos antes da hospitalização e número de medicamentos usados no momento da alta hospitalar).

Houve associação estatisticamente significativa entre os valores dos escores do ICC $(p=0,001)$ e do ICIC $(p=0,001)$ com o desfecho primário da pesquisa (óbito pós-alta hospitalar). Os intervalos interquartis, mediana e valores mínimo e máximo dos índices de comorbidade em relação ao desfecho (óbito) estão apresentados na figura 2 . 


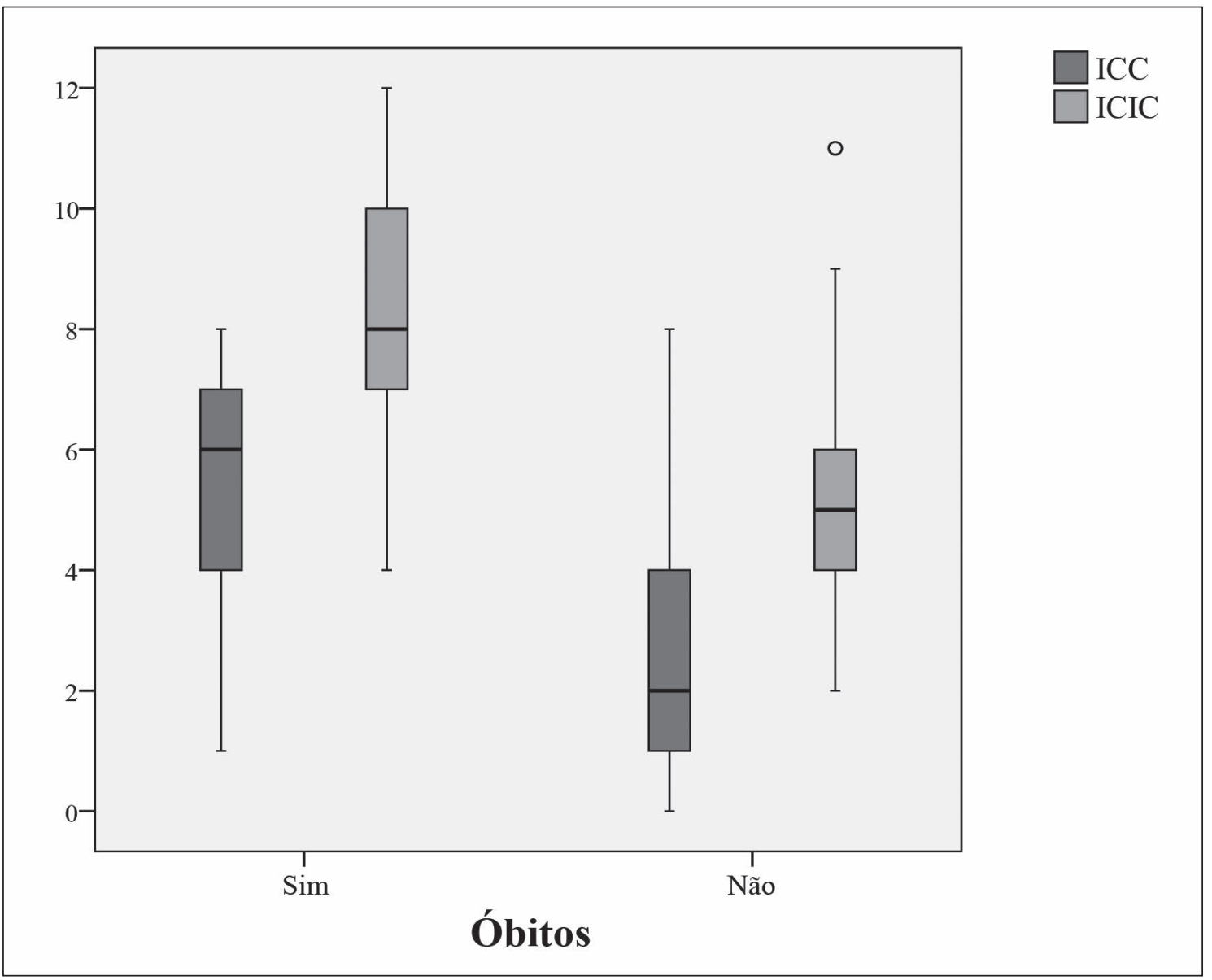

Figura 2 - Diagrama de caixas mostrando medianas, intervalos interquartílicos e valores máximos e mínimos das pontuações do Índice de Comorbidade de Charlston (ICC) e do Índice de Comorbidade de Charlston associado à idade (ICIC), em relação ao desfecho primário do estudo (óbito: sim/não). João Pessoa-PB, 2008-2010.

Pacientes com pontuação de 5 ou mais no ICC apresentaram risco $50 \%$ maior de morte que aqueles com pontuação 4 ou menos (hazard ratio [HR] 1,5 IC 95\% 1,3-1,8; $\mathrm{p}=0,001)$; pacientes com pontuação de 6 ou mais no ICC apresentaram risco de morte $30 \%$ maior que aqueles com pontuação 5 ou menos (haz̧ard ratio [HR] 1,3 1 IC $95 \% 1,2-1,5 ; \mathrm{p}=0,001)$.

Verificou-se associação estatisticamente significativa entre óbito e ICC $(\mathrm{p}=0,002)$, assim como entre óbito e ICIC por meio do teste quiquadrado $(\mathrm{p}=0,001)$. O risco relativo calculado foi 2. Para cada dois óbitos entre os pacientes com
ICC igual ou maior que 5 , houve um óbito entre os pacientes com ICC menor que 5; portanto, verificou-se o dobro (ou 100\% mais) de chances de morrer após a alta hospitalar. Não houve associação estatisticamente significativa entre os valores dos escores do ICC $(p=0,49)$ e do ICIC $(\mathrm{p}=0,55)$ com o desfecho reinternação, como verificado por meio do teste de Mann-Whitney.

Pacientes com pontuação de 5 ou mais no ICC não apresentaram maior risco de reinternação que aqueles com pontuação de 4 ou menos (bazard ratio $[\mathrm{HR}]$ 0,76; IC 95: 0,42-1,39; $\mathrm{p}=0,36$ ). 


\section{DISCUSSÃO}

Os resultados obtidos neste estudo corroboraram parcialmente as hipóteses de pesquisa levantadas: o índice de comorbidades constituiu fator preditivo de mortalidade após alta hospitalar nessa coorte de idosos, mas não se relacionou a um maior risco de reinternação. Há poucos estudos abordando o tema da relação entre comorbidades com o desfecho mortalidade no idoso após internações hospitalares no Brasil, e nenhum deles foi realizado na Região Nordeste do país.

De forma semelhante ao que foi encontrado neste estudo, já foi observado empiricamente que a mortalidade se relaciona com a multimorbidade em idosos, mesmo em contextos diferentes, como ambulatórios e comunidade, porém esta associação declina com o aumento da idade, contrariamente ao que foi encontrado no presente estudo, em que a idade passou a ser um fator importante. ${ }^{21} \mathrm{~A}$ idade da coorte do referido estudo foi muito ampla, desde 50 até 99 anos, e os instrumentos de avaliação das variáveis multimorbidade foram diferentes, explicando possivelmente a diferença encontrada. Além disso, a relação entre mortalidade e comorbidade provavelmente seja mais grave entre os pacientes hospitalizados que seus pares que vivem na comunidade e atendidos ambulatorialmente, sendo esta uma contribuição do presente estudo, uma vez que os trabalhos publicados referem-se a indivíduos em outros contextos.

Resultados semelhantes em relação ao risco relativo foram observados por outros autores, tanto em pacientes idosos ainda hospitalizados, ${ }^{18,22-25}$ quanto em idosos que receberam alta hospitalar. ${ }^{21,26,27}$ As médias do ICC foram semelhantes às encontradas por Cavanaugh et al. ${ }^{25} \mathrm{em}$ idosos internados em um hospital universitário no Sul do Brasil, assim como por Inouye et al. ${ }^{27}$ nos Estados Unidos. Considerando que a prevalência e a complexidade (número de domínios presentes) de comorbidades aumentam com a idade, a taxa encontrada é compatível com a literatura revisada: $83,2 \%$ dos inquiridos em um estudo envolvendo pacientes com 75 anos ou mais tinham multimorbidade. ${ }^{28}$
Não se observou associação entre a contagem simples de comorbidades com mortalidade no presente estudo, diferentemente do que foi encontrado por Newman et al., ${ }^{29}$ mas compatível com o que foi observado por Lee et al. ${ }^{21}$ Esse achado indica que o tipo e a gravidade das comorbidades, conforme indicado pelo ICC, é um fator mais importante que seu número, pela simples contabilização do total de doenças concomitantes.

A taxa de mortalidade encontrada foi semelhante à verificada por outros autores 90 dias após a alta hospitalar dos idosos. ${ }^{27-30}$ Quanto à taxa de reinternação, dados brasileiros indicam que $20,7 \%$ dos idosos têm reinternações no período de um ano após uma alta hospitalar, ${ }^{31}$ taxa semelhante à encontrada no presente estudo. Por outro lado, taxas de readmissão hospitalar foram variáveis em estudos fora do Brasil, como $3,2 \%$ em um mês, ${ }^{30} 10 \%$ em um mês e $23 \%$ em três meses após a alta hospitalar, ${ }^{32}$ de $5 \%$ em um mês e $13 \%$ em um ano. ${ }^{33}$ A maior taxa encontrada foi de $35 \%$ em um ano. ${ }^{34}$

A hospitalização é fator de risco para óbito entre idosos, porque provoca condições de agravo à saúde, como infecções, isolamento social, iatrogenias, entre outras, que podem proporcionar perda de independência e autonomia, muitas vezes levando-os à morte..$^{35,36}$ A variável multimorbidade, por sua vez, é importante agravante durante hospitalizações em pacientes idosos e, posteriormente, na sua sobrevida pós-hospitalização. Esse aspecto é relevante, considerando que em 2003, 14\% (24,6 milhões de pessoas) dos brasileiros idosos foram submetidos a uma ou mais internações hospitalares no ano anterior e, entre estes, 20,7\% tiveram reinternações no mesmo período. ${ }^{31}$ Nesse sentido, sabe-se que as taxas de admissão hospitalar e o tempo médio de ocupação do leito por idosos são significativamente mais elevados quando comparados aos outros grupos etários. ${ }^{37}$

Índices de comorbidade, como ICC, são bons preditores de risco de morte em idosos, já que além de levar em consideração o número de problemas crônicos que sofre um paciente, também incluem a gravidade de cada transtorno no risco de 
morrer. ${ }^{7-36}$ Nas análises de sobrevida, tem-se em conta o tempo de sobrevivência como o período compreendido entre a inclusão de determinado indivíduo no estudo e o aparecimento do desfecho de interesse - neste caso, a morte. Ao se ajustar pela idade, como feito no presente estudo por meio do ICIC, uma vez que outro fator de risco importante para mortalidade continua sendo a própria idade, considera-se que quanto mais se vive, maior é a chance de morrer.

Siqueira et al. ${ }^{35}$ mencionam que a maioria dos estudos longitudinais com idosos residentes na comunidade parece concordar que, além da idade, o sexo do indivíduo pode ser determinante do risco de morte, com os homens apresentando risco maior que as mulheres, o que não foi verificado no presente estudo ou no de Schmidt et al. $^{38}$ É importante considerar, entretanto, que os idosos que não estão hospitalizados encontram-se em condições clínicas diferentes dos pacientes incluídos no presente estudo, mas estes apresentaram resultados compatíveis com aqueles, independentemente do contexto. Contudo, as mulheres apresentaram maior frequência de presença de comorbidades, constituindo uma possível variável explicativa para esse achado na amostra estudada. Também Milcent et al. ${ }^{39}$ verificaram maior frequência de comorbidades em mulheres idosas hospitalizadas que em homens da mesma idade.

As demais variáveis seriam dependentes de uma complexa interação entre o indivíduo e o meio ambiente, que, por sua vez, variaria de cultura para cultura, de uma região para outra do país e também de uma época para outra. Mesmo o fator sexo pode vir a ter sua relação de risco alterada no futuro, com a evolução social promovendo aumento significativo de mortes por doenças cardiovasculares entre as mulheres, agora mais expostas do que antes aos fatores de risco ocupacionais e ambientais. ${ }^{37}$

As doenças mais prevalentes nessa coorte, cardíacas, neoplásicas, pulmonares, renais, acidente vascular cerebral e diabetes mellitus, são as mais fortemente relacionadas com o desfecho morte, o que tem sido relatado por outros autores. ${ }^{1,40}$ A presença de doenças como hipertensão arterial sistêmica, doença hepática crônica e diabetes mellitus são condições associadas com atendimentos clínicos e hospitalizações acima da média no último ano, ${ }^{40}$ embora não tenha sido feita a correlação com o índice de reinternações e mortalidade nesses trabalhos.

A principal limitação para o desenvolvimento deste estudo foi a dificuldade de atender ambulatorialmente todos os participantes da coorte ao longo do seguimento, em virtude da distância geográfica em que se encontravam após a alta hospitalar, realizando-se, nesses casos, o registro do desfecho por contatos telefônicos. Contudo, os desfechos destes pacientes, óbito ou nova hospitalização, eram passíveis de obtenção por meio de comunicação com a família ou com o próprio participante. $\mathrm{E}$ ainda que o estudo tenha seguido modelo observacional analítico, não é possível estabelecer uma relação de causa e efeito entre a gravidade e o tipo de comorbidades avaliados por meio do ICC e o desfecho morte, mas se pode inferir apenas uma associação entre maior ICC e risco de óbito após a hospitalização.

\section{CONCLUSÕES}

Conclui-se que a gravidade e a natureza das comorbidades em idosos que receberam alta das enfermarias de clínica médica do Hospital Universitário Lauro Wanderley relacionaram-se com maior risco de morte pós-hospitalização, sobretudo nos de idade mais avançada. Salientase a importância de estudar a multimorbidade no idoso e a necessidade de se disporem de dados sobre comorbidade para avaliar cuidados de saúde nessa clientela.

Esses resultados são importantes para subsidiar ações de saúde voltadas para idosos egressos de hospitalizações com a finalidade de proporcionar maior vigilância clínica a essa população. Os idosos que apresentam maior risco de óbito devem merecer atenção diferenciada na organização de serviços destinados aos mesmos, o que poderá resultar na diminuição da mortalidade prematura após uma hospitalização. 


\section{REFERÊNCIAS}

1. Martins M, Blais R, Miranda NN. Evaluation of the Charlson comorbidity index among inpatients in Ribeirão Preto, São Paulo State, Brazil. Cad Saúde Pública 2008;24(3):643-52.

2. Kadam UT, Croft PR; North Staffordshire GP Consortium Group. Clinical multimorbidity and physical function in older adults: a record and health status linkage study in general practice. Fam Pract 2007;24(5):412-9.

3. DesHarnais S. Current uses of large data sets to assess the quality of providers. Construction of risk-adjusted indexes of hospital performance. Int J Technol Assess Health Care 1990;6(2):229-38.

4. Rius Gilbert C, Pérez Albarracín G; Grupo Cohesca. La edad como escala de tiempo en el análisis de la supervivencia por trastornos crônicos. Rev Esp Salud Publica 2006;80(6):657-64.

5. Broeiro P, Ramos V, Barroso R. O mapa de problemas: um instrumento para lidar com a morbidade múltipla. Rev Port Clin Geral 2007;23:209-15.

6. Teixeira JJV, Lefèvre F. A prescrição medicamentosa sob a ótica do paciente idoso. Rev Saúde Pública 2001;35(2):207-13.

7. Fortin N, Lapointe L, Hudon C, Vanasse A. Multimorbidity is common to family practice: is it commonly researched? Can Fam Physician $2005 ; 51: 244-5$

8. Starfield B. Global health, equity, and primary care. J Am Board Fam Med 2007;20(6):511-3.

9. Smith SM, Ferede A, O’Dowd T. Multimorbidity in younger deprived patients: an exploratory study of research and service implications in general practice. BMC Fam Pract 2008;9:6.

10. Ministério da Saúde (Brasil). Portaria n. 2.528, de 19 de outubro de 2006. Aprova a Política Nacional de Saúde da Pessoa Idosa [acesso em 21 jan 2012]. Disponível em: http://portal.saude.gov.br/portal/ arquivos/pdf/2528\%20aprova $\% 20 \mathrm{a} \% 20$ politica $\% 20$ nacional $\% 20 \mathrm{de} \% 20$ saude $\% 20 \mathrm{da} \% 20$ pessoa $\% 20$ idosa.pdf

11. Organização Mundial da Saúde. Códigos da Classificação Internacional de Doenças e de Problemas Relacionados a Saúde, Décima Revisão CID-10. São Paulo: EDUSP; 1994.

12. Charlson ME, Pompei P, Ales KL, Mackenzie CR. A new method of classifying prognostic comorbidity in longitudinal studies: development and validation. J Chronic Dis 1987;40(5):373-83.
13. Beloosesky Y, Weiss A, Mansur N. Validity of the Medication-based Disease Burden Index compared with the Charlson Comorbidity Index and the Cumulative Illness Rating Scale for geriatrics: a cohort study. Drugs Aging 2011;28(12):1007-14.

14. Gili M, Sala J, López J, Carrión A, Béjar L, Moreno J, et al. Impact of comorbidities on in-hospital mortality from acute myocardial infarction, 2003-2009. Rev Esp Cardiol 2011;64(12):1130-7.

15. Lemke KW, Weiner JP, Clark JM. Development and validation of a model for predicting inpatient hospitalization. Med Care 2012;50(2):131-9.

16. Martins M. Uso de medidas de comorbidades para predição de risco de óbito em pacientes brasileiros hospitalizados. Rev Saúde Pública 2010;4(3):448-56.

17. Camargo, K.R., Coeli CM. CalcCharlson, versão 1.1 [software na Internet]. 2005 [acesso em 21 jan 2012]. Rio de Janeiro: Universidade do Estado do Rio de Janeiro. Disponível em http://paginas.terra.com.br/ educacao/kencamargo/RecLink.html

18. Incalzi RA, Capparella O, Gemma A, Landi F, Bruno E, Di Meo F, Carbonin P. The interaction between age and comorbidity contributes to predicting the mortality of geriatric patients in the acute-care hospital. J Intern Med 1997;242(4):291-8.

19. Klein JP, Moeschberger ML. Survival Analysis: Techniques for Censored and Truncated Data. New York: Springer; 1997.

20. Bustamante-Teixeira MT, Faerstein E, Latorre MR. Técnicas de análise de sobrevida. Cad Saúde Pública 2002;18(3):579-94.

21. Lee SJ, Go As, Lindquist K, Bertenthal D, Covinsky KE. Chronic conditions and mortality among the oldest old. Am J Public Health 2008;98(7):1209-14.

22. Ramiarina RA, Ramiarina BL, Almeida RMVR, Pereira WCA. Comorbidity adjustment index for the international classification of diseases, 10th revision . Rev Saúde Pública 2008;42(4):590-7.

23. Maia FOM, Duarte YAO, Lebrão ML, Santos JLF. Fatores de risco para mortalidade em idosos. Rev Saúde Pública 2006;40(6):1049-56.

24. Iucif Jr N, Rocha JSY. Estudo da desigualdade na mortalidade hospitalar pelo índice de comorbidade de Charlson. Rev Saúde Pública 2004;38(6):780-6.

25. von Ammon Cavanaugh S, Furlanetto LM, Creech SD, Powell LH. Medical illness, past depression, and present depression: a predictive triad for in-hospital mortality. Am J Psychiatry 2001;158(1):43-8. 
26. Rozzini R, Frisoni GB, Ferrucci L, Barbisoni P, Sabatini T, Ranieri P, et al. Geriatric index of comorbidity validation and comparison with other measures of comorbidity. Age Ageing 2002;31(4):277-85.

27. Inouye SK, Zhang Y, Jones RN, Shi P, Cupples La, Calderon $\mathrm{HN}$, et al. Risk factors for hospitalization among community-dwelling primary care older patients: development and validation of a predictive model. Med Care 2008;46(7):726-31.

28. Britt HC, Harrison CM, Miller GC, Knox SA. Prevalence and patterns of multimorbidity in Australia. Med J Aust 2008;189(2):72-7.

29. Newman AB, Boudreau RM, Naydeck BL, Fried LF, Harris TB. A physiologic index of comorbidity: relationship to mortality and disability. J Gerontol A Biol Sci Med Sci 2008;63(6):603-9.

30. Zanocchi M, Maero B, Martinelli E, Cerrato F, Corsinovi L, Gonella M, et al. Early re-hospitalization of elderly people discharged from a geriatric ward. Aging Clin Exp Res 2006;18(1):63-9.

31. Instituto Brasileiro de Geografia e Estatística. Doenças crônicas atingem quase um terço da população brasileira. 2003 [acesso em 21 jan 2012]. Disponível em: http://www.ibge.gov.br/home/ presidencia/noticias/noticia_visualiza.php?id_ noticia=370\&id_pagina=1. Acesso: 16 fev 2012.

32. Comette P, D'Hoore W, Malhomme B, Van Pee D, Meert P, Swine C. Differential risk factors for early and later hospital readmission of older patients. Aging Clin Exp Res 2005;17(4):322-8.

33. Halfon P, Eggli Y, van Melle G, Chevalier J, Wasserfallen JB, Burnand B. Measuring potentially avoidable hospital readmissions. J Clin Epidemiol 2002;55(6):573-87.

34. Buurman BM, Hoogerduijn JG, de Haan RJ, AbuHanna A, Lagaay AM, Verhaar HJ, et al. Geriatric conditions in acutely hospitalized older patients: prevalence and one-year survival and functional decline. PLoS One 2011;6(11):e26951.

35. Siqueira AB, Cordeiro RC, Perracini MR, Ramos LR.. Impacto funcional da internação hospitalar de pacientes idosos. Rev Saúde Pública 2004;38(5):687-94.

36. Smyth C, Dubin S, Restrepo A, Nueva-Espana H, Capezuti E. Creating order out of chaos: models of GNP practice with hospitalized older adults. Clin Excell Nurse Pract 2001;5(2):88-95.

37. Amaral ACS, Coeli CM, Costa MCE, Cardoso VS, Toledo ALA, Fernandes CR. Perfil de morbidade e de mortalidade de pacientes idosos hospitalizados. Cad Saúde Pública 2004;20(6):1617-26.

38. Schmidt M, Jacobsen JB, Lash TL, Botker HE, Sorensen HT. 25 year trends in first time hospitalisation for acute myocardial infarction, subsequent short and long term mortality, and the prognostic impact of sex and comorbidity: a Danish nationwide cohort study. BMJ 2012;344:e356.

39. Milcent C, Dormont B, Durand-Zaleski I, Steg PG. Gender differences in hospital mortality and use of percutaneous coronary intervention in acute myocardial infarction: microsimulation analysis of the 1999 nationwide French hospitals database. Circulation 2007;115(7):833-9.

40. Alvarenga MRM, Mendes MMR. El perfil de los reingresos de ancianos en un hospital general del municipio de Marília, SP. Rev Latino-Am Enferm 2003;11(3):305-11. 